\title{
Somatostatin effects on the proteome of the LNCaP cell-line
}

\author{
ZHAOXU LIU $^{1,5}$, SOFIA BENGTSSON ${ }^{2}$, MORTEN KROGH ${ }^{3}$, MARCELA MARQUEZ ${ }^{1}$, \\ STEN NILSSON $^{1}$, PETER JAMES ${ }^{2}$, AYODELE ALIAYA ${ }^{4}$ and ANDERS R. HOLMBERG ${ }^{1}$ \\ ${ }^{1}$ Department of Oncology and Pathology, Karolinska Institute, SE-171 76 Stockholm; ${ }^{2}$ Department of Protein Technology, \\ ${ }^{3}$ Complex Systems Division, Department of Theoretical Physics, Lund University, Lund SE-22100, Sweden; \\ ${ }^{4}$ Department of Biological and Medical Research, King Faisal Specialist Hospital, 11211 Riyadh, Saudi Arabia; \\ ${ }^{5}$ Aging and Health Center, School of Nursing/Qilu Hospital, Shandong University, 250012 Jinan, P.R. China
}

Received October 19, 2006; Accepted December 1, 2006

\begin{abstract}
Some clinical results indicate that somatostatin (sms) might be useful in the treatment of advanced prostate cancer (HRPC). Because of its transient in vivo half-life only more stable derivatives of sms are of interest in this context. Recent studies have shown that natural sms can be conjugated to a carbohydrate ( $\operatorname{smsdx}$ ) with preservation of sms-like effects on the prostatic tumor cell proteome. The present study identifies some of the affected proteins in an effort to elucidate pathways and proteins that might be of importance for the potential usefulness of sms treatment in HRPC. After incubating the LNCaP cell-line with sms14/smsdx, comparative proteomics was used for analysing and identifying affected proteins. Protein expression patterns were analysed with two-dimensional polyacrylamide gel electrophoresis and mass spectrometry. Catalytic mitochondrial and mitochondrial-associated proteins were significantly affected (fold change $\sim 2$ or higher) and they were in general up-regulated. Apoptosis-related proteins were both up-regulated (VDAC1, VDAC2) and down-regulated (PRDX2, TCTP). The fold change was $>2$ for PRDX2 and $<2$ for the others. There was a strong agreement between sms and smsdx on the up- and down-regulation of proteins. Sms/smsdx triggered up-regulation of catalytic mitochondrial proteins and seemed to affect apoptosis-related proteins. This could indicate important pathways on which smsdx might be able to curb the progression of HRPC. The results encourage a pending clinical phase II study.
\end{abstract}

\section{Introduction}

Prostate cancer is a major health problem in the Western world being the second leading cause of cancer related death

Correspondence to: Dr Anders R. Holmberg, CCK R8/3, Urologic Oncology Group, Karolinska University Hospital, Karolinska Institute, SE-171 76 Stockholm, Sweden

E-mail: arh@telia.com

Key words: two-dimensional electrophoresis, mass spectrometry, prostatic cancer, $\mathrm{LNCaP}$, protein expression, somatostatin in men (1). The prognosis of early-stage well-differentiated prostate cancer is favourable however some patients have extra-prostatic invasion at the time of diagnosis and some of these have metastatic disease (2). In early-stage localised disease, radical prostatectomy or radiation therapy, or a combination of both modalities, are considered curative. In metastatic disease androgen-ablation therapy is standard treatment, however the disease progresses eventually and becomes unresponsive to hormonal treatment (hormone refractory prostatic cancer, HRPC). At this stage few treatment options exist and new modalities are urgently needed. Recently, a taxan derivative has shown some treatment efficacy in HRPC (3). Additionally, recent results with sms derivatives combined with dexamethasone or in combination with estrogens seem promising (4).

Somatostatin (sms) is important in the regulation and physiological control of various organs including the prostate. Sms can inhibit tumor growth (growth arrest), be cytotoxic (induction of apoptosis) and inhibit tumor angiogenesis $(5,6)$. The sms analog Octreotide inhibits the growth of MCF-7 by activating wt p53 and Bax and triggers rapid apoptosis in this cell-line (7). Sms receptors (SSTRs) might be potential targets for prostate cancer treatment. However, optimal dose/ dose interval and the optimal sms derivative still need to be determined.

Recently an sms derivative ( $\operatorname{smsdx}$ ) based on natural sms 14 having affinity to all five SSTR subtypes, was developed $(8,9)$. The long half-life and the pan affinity of smsdx distinguish it from all other sms derivatives in clinical use which have shorter half-lives and limited SSTR affinity (affinity only to SSTR2 and 5). Smsdx has completed a clinical phase I study revealing low toxicity and high tolerability (10).

LNCaP is a human prostate cancer cell-line and often used in various in vitro studies. It has been established from supraclavicular lymph node metastases (11). These cells express androgen receptors (ARs) and exhibit androgen-sensitive cell growth. Although there is a physiologically equivalent level of androgenic activity in the regular culture condition, $\mathrm{LNCaP}$ cells gradually lose their androgen requirement for growth upon passage, mimicking tumor progression in patients (12-14).

In a recent study, it was demonstrated that smsdx affect the proteome of $\mathrm{LNCaP}$ in an sms14-like way i.e. sms 14 effects are preserved in the construct (15). In the present study, 
alterations in protein expression patterns of $\mathrm{LNCaP}$ after incubation with sms/smsdx were analysed. Two-dimensional gel electrophoresis (2DE) in combination with mass spectrometry was used for the identification of differentially affected proteins. The character of some of the proteins and their possible role in how sms might be able to curb the progression of HRPC were elucidated.

\section{Materials and methods}

Cell line culture. LNCaP human prostate cancer cell line (American Type Culture Collection, Rockville, MD, USA) was cultured in RPMI-1640 containing 10\% fetal bovine serum (FBS), $2 \mathrm{mM}$ glutamine and $100 \mathrm{IU} / \mathrm{ml}$ penicillin- $100 \mu \mathrm{g} / \mathrm{ml}$ streptomycin at $37^{\circ} \mathrm{C}$ in a humidified atmosphere of $5 \% \mathrm{CO}_{2}$. The medium was changed twice a week and the cells were trypsinized and subcultivated once a week. Smsdx was prepared as described previously (9). Sms was from Ferring, Kiel, Germany. The cell culture was treated with smsdx or with sms for 3 days, $1 \mathrm{nM}$ per day, as described by Brevini et al (16). Controls were untreated cells (negative) and cells treated with sms (positive).

Sample preparation for $2 D-P A G E$. The cellular extraction from $\mathrm{LNCaP}$ and the preparation of the total cell lysate were performed as previously described by Franzen et al (17). Protein determination was made using Pierce BCA protein assay reagent (Rockford, IL, USA). Protein (500 $\mu \mathrm{g}$ ) was mixed with rehydration buffer [8 $\mathrm{M}$ urea, 2\% CHAPS, $0.002 \%$ bromophenol blue, $18.2 \mathrm{mM}$ DTT, $0.5 \%$ pharmalyte $(\mathrm{pH} 3-10$ $\mathrm{NL})$ ], left at room temperature for $15 \mathrm{~min}$ and centrifuged for $10 \mathrm{~min}$ before being applied to a $24-\mathrm{cm}$ immobilized $\mathrm{pH}$ gradient strip ( $\mathrm{pH}$ 3-10 NL) for overnight rehydration.

2D-PAGE and image analysis. First-dimension isoelectric focusing was carried out on GE Healthcare IPG-phor according to the manufacturer's instructions, with a total focusing time of around $70 \mathrm{kVh}$. The strips were equilibrated in $15 \mathrm{ml}$ equilibration solution [6 $\mathrm{M}$ urea, $50 \mathrm{mM}$ Tris- $\mathrm{HCl}(\mathrm{pH} 8.8)$, $30 \%(\mathrm{w} / \mathrm{v})$ glycerol, $2 \%(\mathrm{w} / \mathrm{v})$ SDS, $0.002 \%$ bromophenol blue] reduced with $65 \mathrm{mM}$ DTT for $15 \mathrm{~min}$, followed by 15-min equilibration in equilibration solution with $135 \mathrm{mM}$ iodoacetamide added. The IPG-strips were then loaded and run on a $12.5 \%$ SDS-PAGE gel $\left(25^{\circ} \mathrm{C}\right)$ with $10 \mathrm{~W} /$ gel until the bromophenol blue dye front had run off the base of the gel. The gels were stained in colloidal coomassie (LabDesign Boule Nordic $\mathrm{AB}$ ) overnight and then destained in water according to the manufacturer's instructions. The gels were scanned using GE Healthcare ImageScanner. Spot detection and matching was performed in ImageMaster 2D Platinum (GE Healthcare) with three manual landmarks set.

Statistical analysis. Three gels were run for each of the three groups; control, smsdx, and sms. The master gel belonged to the smsdx group. The 1083 spots on the master gel were matched with spots on the other 8 gels, when possible. If no matching spot was found on a gel, it was designated as a missing value. A spot can be missing either due to an experimental error or due to low abundance. The two treated groups, sms and smsdx, were compared separately with the control group using a Mann-Whitney test. Since the control group and a treated group contains three measurements each, the lowest possible two-sided Mann-Whitney p-value is 0.1 , which is achieved exactly when all 3 values in one group are lower or higher than all 3 values in the other group. This can only happen when there are no missing values. The number of spots without any missing values in the smsdx and control group was 468 . Out of these, 137 had a p-value of 0.1 , leading to a false discovery rate of 0.29 . To include the rest of the spots, missing values were set to zero in the Mann-Whitney test. So, the spots with a p-value of 0.1 were those with the three highest measurements in one group, and all measurements in the other group were either lower or missing. The number of spots in the control vs smsdx comparison with at least one missing value was 615 . Out of these, 100 had a p-value of 0.1 , leading to a false discovery rate of 0.62 . The total number of spots with a p-value of 0.1 for the control vs. smsdx comparison was hence 237 out of 1083 , leading to a false discovery rate of 0.46 . For the control vs. the sms group, the corresponding numbers were: 140 out of $436($ FDR $=0.31)$ without missing values, 83 out of $647($ FDR $=0.78)$ with missing values, and 223 out of 1083 (FDR $=0.49$ ) in total.

Mass spectrometry analysis. Proteins were identified with a vMALDI-LTQ instrument (Thermo Electron, San José, CA). The spot picking, destaining, digestion, extraction, sample preparation and spotting on MALDI target plates were carried out using a spothandling workstation (ETTAN Spothandling workstation, GE Healthcare) and a standard protocol provided by GE Healthcare. The plate containing the combined extracts was evaporated to dryness. Each sample was prepared by constituting the dried peptides in $2.5 \mu 1$ of matrix solution [2.5 mg/ml of $\alpha$-cyano-4-hydroxy-cinnamic acid (Sigma) in $50 \%$ acetonitrile containing $0.05 \%$ TFA]. Sample $(2.0 \mu 1)$ was spotted on a clean MALDI target slide surface and allowed to dry. The samples were analysed with a vMALDILTQ (Thermo Electron). The analysis was performed using Xcalibur 1.4 software in data dependent mode. A survey scan (MS) was followed by MS/MS scans on the 5 most abundant ions. This string of 6 scan events was repeated six times for each sample spot. Dynamic Exclusion ${ }^{\mathrm{TM}}$ ensured that in total 30 different peptides were selected and fragmented for each sample. The MS spectra were collected in the 900-2.000 Da mass range while the mass range for the MS/MS spectra was automatically selected by the system based on a $\mathrm{Q}$ value of 0.25 . The standard collision energy of 38 was set for all analyses. A time limit of $5 \mathrm{~min} / \mathrm{sample}$ was selected, whether or not the $30 \mathrm{MS} / \mathrm{MS}$ spectra could be acquired. Database searches were performed using both the Mascot and Sequest search algorithm against the human session of the IPI protein database (version 2.38). The two searches were compared in the in-house developed software Promiscuous MS/MS. A minimum of two peptides and a Mascot score of 45 were required for a protein to be accepted as identified.

\section{Results}

Functional classification of proteins. The proteins were classified using the Swiss-Prot website, the Genome Browser website, and the National Center for Biotechnology Information 
A

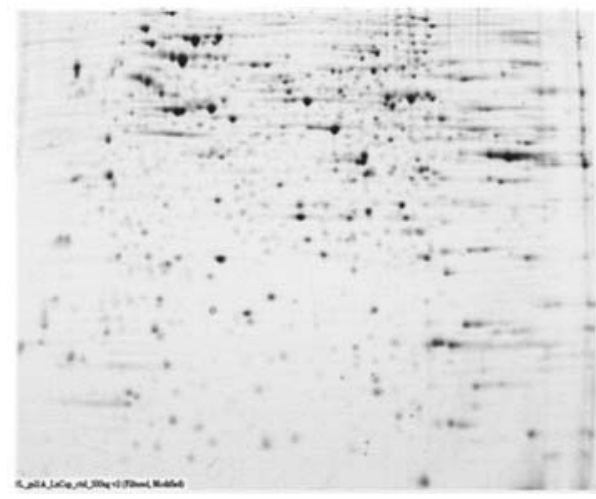

B

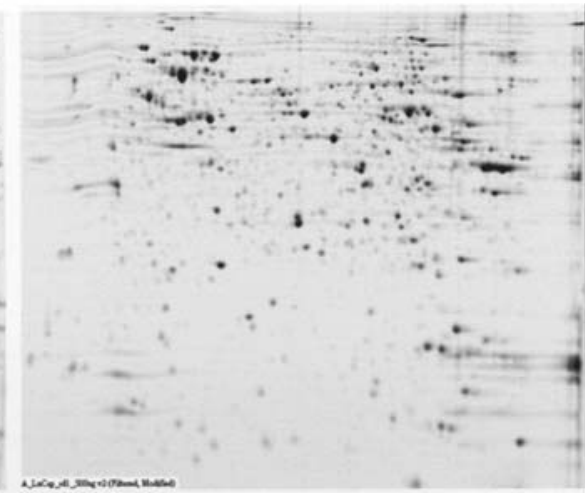

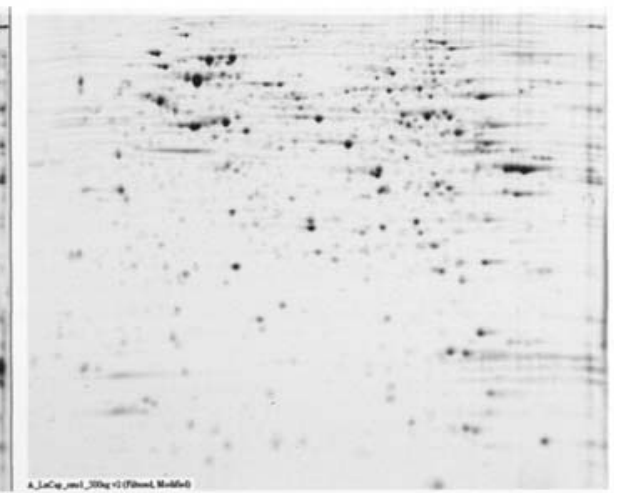

Figure 1. Representative examples of 2-DE gels derived from control, smsdx and sms treated prostate cancer cell line LNCaP. Whole-cell lysate was subjected to 2-DE using IPG strips pH 3-10 in the first and 12.5\% SDS polyacrylamide gel in the second dimension.

(NCBI) Locuslink website. They were categorized on the basis of their known biochemical functions including metabolism, signalling transduction, maintenance of cell structure, cell-cell interactions, transport/trafficking, transcriptional regulation and cell cycle regulation

2D gel electrophoresis analysis. Fig. 1 shows representative 2D gels of control, sms and smsdx samples. Three replicates were run for each group, control, sms and smsdx. The sms and smsdx were compared separately with the control group using a Mann-Whitney test. A total of 172 spots were identified using MALDI-MS/MS, 140 different proteins were identified and consequently the same protein was sometimes found in multiple spots.

Sms/smsdx affected proteins. Proteins affected by sms/smsdx incubation with a fold change $\geq 1.6$ and a Mann-Whitney p-value of 0.1 when discarding missing values are listed in Table I. Several of these were catalytic mitochondrial proteins and they were usually up-regulated, e.g. ACO2, PNPTT1, ACADS, only isocitrate dehydrogenase (NAD) subunit $\alpha$ was down-regulated. One protein, PRDX2, was apoptosis related and was down-regulated. Other proteins affected by sms/smsdx are listed in Table II (fold change $<1,6$ ). For significantly affected proteins, there was a clear agreement in the up- and down-regulation between sms and smsdx (Tables I and II).

\section{Discussion}

Somatostatin has an important role in biological processes including neurotransmission, exocrine/endocrine secretion, and cell proliferation. It acts via a family of five GCPRs (G-protein-coupled receptors), i.e. SSTR1-SSTR5. Its antiproliferative action results either from inhibition of trophic or growth factor secretion or from interference with the cell cycle progression (18). These properties make sms derivatives interesting drug candidates for the treatment of cancers expressing SSTRs especially those where efficacious treatment options are missing as in the case of HRPC. In this study, several mitochondrial proteins and apoptosis/cancer related proteins were identified. There was a strong correlation between sms and smsdx concerning the up- and downregulation of the proteins. Additionally, the regulation was in general as could be expected (up or down depending on function). The mitochondria plays a key role in many cellular functions including energy production, fatty acid metabolism, pyrimidine biosynthesis, calcium homeostasis, and cell signalling. It serves as a control point in regulating the intrinsic and extrinsic apoptotic pathway in response to DNA damage. Apoptotic cell death can be induced by a number of triggers released from the mitochondrial outer membrane (e.g. cytochrome c, Smac/Diablo, and apoptosis-inducing factor, AIF, caspase-dependent/independent pathways). The release can be blocked by overexpression of Bcl-2 (19). Mitochondria is important in the control of cell survival and death (20) and is an important target for anticancer chemotherapy (21). Several of the proteins involved in the MMP event (mitochondrial membrane permeabilization proteins), e.g. VDAC, IDH3A, and NADP, were identified. MMP is a critical event in the process leading to chemotherapy induced apoptosis. The permeabilization is controlled by the permeability transition pore complex (PTPC). Bax can associate with the voltage dependant anion channel (VDAC), which is also located in the outer mitochondrial membrane. The VDAC-Bax complex promotes pore formation, outer MMP, and the release of AMPs to activate caspases and induce apoptosis. Bcl-2 family proteins also regulate the release of apoptogenic cytochrome $\mathrm{c}$ by the mitochondrial channel VDAC (22). VDAC1 has been shown to coimmuneprecipitate with the anti-apoptotic protein $\mathrm{Bcl}$ and suggested to be involved in forming the mitochondrial pore which releases cytochrome $\mathrm{c}$ during apoptosis (23). From the present results it seems reasonable to assume that sms/ smsdx can affect the mitochondria of $\mathrm{LNCaP}$ in a way that eventually could trigger mitochondrial-mediated apoptosis.

The regulation of peroxiredoxins (PRDX) is of importance for apoptosis and cell proliferation $(24,25)$. PRDXs can be regulated by changes in phosphorylation, redox and possibly by oligomerization states. It appears that high levels of PRDXs inhibit apoptosis and induce cell proliferation (26). PRDX2 prevents cytochrome c release from mitochondria and inhibits lipid peroxidation. Suppression of PRDX2 is 
Table I. Proteins with strong differential expression ( $>1.6$ in fold change) between smsdx vs. control and sms vs. control.

\begin{tabular}{llcccc}
\hline Protein name & $\begin{array}{c}\text { Short } \\
\text { name }\end{array}$ & $\begin{array}{c}\text { UniProt } \\
\text { ID }\end{array}$ & $\begin{array}{c}\text { Fold change } \\
\text { smsdx vs. ctrl }\end{array}$ & $\begin{array}{c}\text { Fold change } \\
\text { sms vs. ctrl }\end{array}$ & $\begin{array}{c}\text { Trends } \\
\text { sms/smsdx vs. ctrl }\end{array}$ \\
\hline
\end{tabular}

\section{Apoptosis}

Voltage-dependent anion channel 1

VDAC1 P21796

DNA binding

TATA-binding protein associated factor $2 \mathrm{~N}$

Heterogeneous nuclear ribonucleoprotein D0

\section{Electron transport}

Protein disulfide-isomerase

Ubiquinol-cytochrome c reductase

iron-sulfur subunit, mitochondrial

Metabolism

Short chain 3-hydroxyacyl-CoA

dehydrogenase, mitochondrial

Glycerol-3 phosphate dehydrogenase, mitochondrial

Acetyl-CoA acetyltransferase cytosolic

Isocitrate dehydrogenase (NAD)

subunit $\alpha$ mitochondrial

Aconitate hydratase, mitochondrial

Proliferation

Peroxiredoxin 2

Heterogeneous nuclear ribonucleoproteins C1/C2

Protein binding

Early endosome antigen 1

Lamin A/C

\begin{tabular}{|c|c|c|c|c|}
\hline $\mathrm{HCDH}$ & Q16836 & 1.66 & 1.68 & sms $/$ smsdx $>$ ctrl \\
\hline GPDM & P43304 & 1.62 & 1.75 & sms/smsdx $>$ ctrl \\
\hline THIC & Q9BWD1 & Inf & Inf & unique to $\mathrm{sms} / \mathrm{smsdx}$ \\
\hline IDH3A & P50213 & -1.78 & -1.72 & $\operatorname{ctrl}>\operatorname{sms} /$ smsdx \\
\hline $\mathrm{ACON}$ & Q99798 & 2.50 & 2.17 & sms/smsdx $>$ ctrl \\
\hline PRDX2 & P32119 & -2.06 & -2.14 & ctrl $>$ sms/smsdx \\
\hline HNRPC & P07910 & 2.56 & 2.65 & sms $/$ smsdx $>$ ctrl \\
\hline EEA1 & Q15075 & Inf & Inf & unique to $\mathrm{sms} / \mathrm{smsdx}$ \\
\hline LMNA & P02545 & Inf & Inf & unique to sms/smsdx \\
\hline $\mathrm{CBX} 3$ & Q13185 & Inf & Inf & unique to sms/smsdx \\
\hline TIF1B & Q13263 & Inf & Inf & unique to $\mathrm{sms} / \mathrm{smsdx}$ \\
\hline VDAC2 & P45880 & 1.84 & 1.79 & sms $/$ smsdx $>$ ctrl \\
\hline
\end{tabular}

No missing values and the lowest possible two-sided Mann-Whitney p-value (=0.1). Protein accession numbers from SwissProt/TrEMBL are given and 'trend' indicates the direction of the change in expression level. The proteins are grouped according to molecular function. Smsdx and sms appear to have a similar effect demonstrated by the concordance in protein expression levels. Ctrl, control.

associated with enhancement of radiation sensitivity (27). In the present study, sms/smsdx down-regulated PRDX2.

The heat-shock protein HSPB 1 was affected by sms/ smsdx. However, the regulation was different between sms and smsdx. Sms down-regulated while smsdx up-regulated (fold change $<1,6$ ). In advanced cancer, HSP27 expression is associated with poor clinical outcome. HSP70, like HSP27, protects cells from stress-induced caspase-dependent apoptosis, both upstream and downstream of the apoptosis associated mitochondrial events (28). Evidence for signalling and pathways leading to changes in the cancer cells apoptotic threshold is scarce. If anticancer drugs are administered at suboptimal concentrations, i.e. to trigger apoptosis, therapy resistance may actually be enhanced through up-regulation of anti-apoptotic factors such as HSP27 (29). Consequently, the optimal dosage of sms derivatives needs to be explored and determined to elucidate its clinical usefulness. The discrepancy seen between sms and smsdx may be related with the appropriate dose, i.e. smsdx might be needed in a higher concentration to have a similar effect on HSPB1 as sms. The chaperone protein, GRP78, was up-regulated by both sms and smsdx.

GRP78 is expressed on the cell surface of prostate cancer cells and appears to mediate the signal transduction of B2macroglobulin $(30,31)$. It correlates with the development of androgen-independent disease and shorter overall survival in prostate cancer patients. Arap et al (32) have recently identified GRP78 as a potential molecular target that may prove useful for translation into clinical applications.

Stathmin, a family of microtubule-destabilizing proteins, was down-regulated by $\mathrm{sms} / \mathrm{smsdx}$. It plays a critical role 
Table II. Proteins with no missing values and a two-sided Mann-Whitney p-value of 0.1 when comparing smsdx vs. control and sms vs. control.

\begin{tabular}{lccccc}
\hline Protein name & $\begin{array}{c}\text { Short } \\
\text { name }\end{array}$ & $\begin{array}{c}\text { UniProt } \\
\text { ID }\end{array}$ & $\begin{array}{c}\text { Fold change } \\
\text { smsdx vs. ctrl }\end{array}$ & $\begin{array}{c}\text { Fold change } \\
\text { sms vs.ctrl }\end{array}$ & $\begin{array}{c}\text { Trends } \\
\text { sms/smsdx vs.ctrl }\end{array}$ \\
\hline
\end{tabular}

Antioxidant activity

Superoxide dismutase 1 , soluble

Peroxiredoxin 6

ATP binding

Stress-70 protein

Glutamate dehydrogenase 1

Glutathione synthetase

Nucleoside diphosphate kinase A

DNA binding

TATA-binding protein associated factor $2 \mathrm{~N}$

Heterogeneous nuclear ribonucleoprotein $\mathrm{K}$

Heterogeneous nuclear ribonucleoprotein D0

Paraspeckle protein 1

ER activity

SAPK substrate protein 1

FAD binding

Dihydrolipoyl dehydrogenase

Acyl CoA dehydrogenase, medium-chain specific

Elecron transfer flavoprotein $\alpha$-subunit, mitochondrial

GTP binding

Tubulin $\beta$ chain

Metabolism

Inorganic pyrophosphatase

Esterase D/formylglutathione hydrolase

Triosephosphate isomerase

Triosephosphate isomerase

Adenine phosphoribosyltransferase

Aconitate hydratase

D-3-phosphoglycerate dehydrogenase

D-3-phosphoglycerate dehydrogenase

Lipoamide acyltransferase component of branched-

chain $\alpha$-keto acid dehydrogenase complex

Fructose-bisphosphate aldolase $\mathrm{C}$

3-hydroxyisobutyryl-Coenzyme A hydrolase

Delta3.5-delta2.4-dienoyl-CoA isomerase

3-hydroxyisobutyrate dehydrogenase, mitochondrial

$\begin{array}{lllll}\text { SODC } & \text { P00441 } & -1.3781 & -1.4002 & \text { ctrl }>\text { sms/smsdx } \\ \text { PRDX6 } & \text { P30041 } & -1.2558 & -1.2730 & \text { ctrl }>\text { sms/smsdx }\end{array}$

$\begin{array}{llrrl}\text { GRP75 } & \text { P38646 } & -1.3487 & 1.3941 & \text { sms } / \text { smsdx }>\text { ctrl } \\ \text { DHE3 } & \text { P00367 } & -1.3958 & 1.4294 & \text { sms } / \text { smsdx }>\text { ctrl } \\ \text { GSHB } & \text { P48637 } & -1.5522 & -1.6268 & \text { ctrl }>\text { sms } / \text { smsdx } \\ \text { NDKA } & \text { P15531 } & -1.3200 & -1.4463 & \text { ctrl }>\text { sms } / \text { smsdx }\end{array}$

$\begin{array}{lllll}\text { RBP56 } & \text { Q92804 } & 1.6136 & 1.4939 & \text { sms } / \text { smsdx }>\text { ctrl } \\ \text { HNRPK } & \text { P61978 } & 1.2153 & 1.3092 & \text { sms } / \text { smsdx }>\text { ctrl } \\ \text { HNRPD } & \text { Q14103 } & 1.4724 & 1.3167 & \text { sms } / \text { smsdx }>\text { ctrl } \\ \text { Q8WXF1 } & \text { Q8WXF1 } & 1.3522 & 1.3365 & \text { sms } / \text { smsdx }>\text { ctrl }\end{array}$

SAKS1 Q04323 $\quad-2.0809 \quad-1.3321 \quad$ ctrl $>$ sms $/$ smsdx

$\begin{array}{lllll}\text { DLDH } & \text { P09622 } & 1.6057 & 1.5586 & \text { sms } / \text { smsdx }>\text { ctrl } \\ \text { ACADM } & \text { P11310 } & 1.5840 & 1.4986 & \text { sms } / \text { smsdx }>\text { ctrl } \\ \text { ETFA } & \text { P13804 } & 1.4264 & 1.4131 & \text { sms } / \text { smsdx }>\text { ctrl }\end{array}$

$\begin{array}{lllll}\text { TBB2C } & \text { P68371 } & -1.2300 & -1.4093 \quad \text { ctrl }>\text { sms } / \text { smsdx }\end{array}$

$\begin{array}{llrrl}\text { IPYR } & \text { Q15181 } & -1.3124 & -1.3867 & \mathrm{ctrl}>\mathrm{sms} / \mathrm{smsdx} \\ \text { ESTD } & \text { Q9BVJ2 } & -1.2460 & -1.2094 & \mathrm{ctrl}>\mathrm{sms} / \mathrm{smsdx} \\ \text { TPIS } & \text { P60174 } & -1.2045 & -1.2217 & \mathrm{ctrl}>\mathrm{sms} / \mathrm{smsdx} \\ \text { TPIS } & \text { P60174 } & -1.3283 & -1.2866 & \mathrm{ctrl}>\mathrm{sms} / \mathrm{smsdx} \\ \text { APT } & \text { P07741 } & -1.2496 & -1.1947 & \mathrm{ctrl}>\mathrm{sms} / \mathrm{smsdx} \\ \text { ACON } & \text { Q99798 } & 1.3559 & 1.3665 & \mathrm{sms} / \mathrm{smsdx}>\mathrm{ctrl} \\ \text { SERA } & \text { O43175 } & -1.3422 & -1.4193 & \mathrm{ctrl}>\mathrm{sms} / \mathrm{smsdx} \\ \text { SERA } & \text { O43175 } & -1.2660 & -1.1457 & \mathrm{ctrl}>\mathrm{sms} / \mathrm{smsdx} \\ \text { ODB2 } & \text { P11182 } & 1.5934 & 1.6911 & \mathrm{sms} / \mathrm{smsdx}>\mathrm{ctrl} \\ & & & & \\ \text { ALDOC } & \text { P09972 } & 1.4677 & 1.3982 & \mathrm{sms} / \mathrm{smsdx}>\mathrm{ctrl} \\ \text { Q53GF2 } & \text { Q53GF2 } & 1.4179 & 1.2932 & \mathrm{sms} / \mathrm{smsdx}>\mathrm{ctrl} \\ \text { ECH1 } & \text { Q13011 } & 1.1811 & 1.2482 & \mathrm{sms} / \mathrm{smsdx}>\mathrm{ctrl} \\ \text { 3HIDH } & \text { P31937 } & 1.2684 & 1.2447 & \mathrm{sms} / \mathrm{smsdx}>\mathrm{ctrl}\end{array}$

Proliferation

$78 \mathrm{kDa}$ glucose-regulated protein

$\begin{array}{llr}\text { GRP78 } & \text { P11021 } & 1.3518 \\ \text { TCTP } & \text { P13693 } & -1.4436 \\ \text { GDIR } & \text { P52565 } & -1.4795\end{array}$

1.4180

$-1.5709$

Translationally controlled tumor protein

Rho GDP-dissociation inhibitor 1

GDIR

P52565

$-1.4795$

Protein binding

Keratin, type II cytoskeletal 1

Heat-shock protein $\beta-1$

$\begin{array}{llr}\text { K2C1 } & \text { P04264 } & 1.4002 \\ \text { HSPB1 } & \text { P04792 } & 1.7470 \\ & & \\ \text { LA } & \text { P05455 } & -1.5795 \\ \text { HNRPL } & \text { P14866 } & 1.2634 \\ \text { HNRPL } & \text { P14866 } & 1.3782 \\ \text { HNRPH } & \text { P31943 } & 1.2937\end{array}$

1.2694

$-1.3354$

smsdx $>$ ctrl $>$ sms

RNA binding

Lupus La protein

Heterogeneous nuclear ribonucleoprotein L

Heterogeneous nuclear ribonucleoprotein L

Heterogeneous nuclear ribonucleoprotein $\mathrm{H}$
HNRPH P31943 1.2937 sms/smsdx $>$ ctrl

sms/smsdx $>$ ctrl ctrl $>$ sms/smsdx ctrl $>$ sms/smsdx

ctrl $>$ sms/smsdx sms/smsdx $>$ ctrl sms/smsdx $>$ ctrl sms/smsdx $>$ ctrl 
Table II. Continued.

\begin{tabular}{|c|c|c|c|c|c|}
\hline Protein name & $\begin{array}{l}\text { Short } \\
\text { name }\end{array}$ & $\begin{array}{l}\text { UniProt } \\
\text { ID }\end{array}$ & $\begin{array}{l}\text { Fold change } \\
\text { smsdx vs. ctrl }\end{array}$ & $\begin{array}{l}\text { Fold change } \\
\text { sms vs. ctrl }\end{array}$ & $\begin{array}{c}\text { Trends } \\
\text { sms/smsdx vs.ctrl }\end{array}$ \\
\hline \multicolumn{6}{|l|}{ RNA binding } \\
\hline Cleavage stimulation factor, $50 \mathrm{kDa}$ subunit & CSTF1 & Q05048 & 1.1899 & 1.2589 & sms/smsdx $>$ ctrl \\
\hline Proteasome subunit $\alpha$ type 1 & PSA1 & P25786 & -1.3587 & -1.4821 & $\operatorname{ctrl}>\operatorname{sms} / \mathrm{smsdx}$ \\
\hline \multicolumn{6}{|l|}{ RNA splicing } \\
\hline Far upstream element binding protein 2 & FUBP2 & Q92945 & 1.3225 & 1.2989 & sms/smsdx $>$ ctrl \\
\hline Far upstream element binding protein 2 & FUBP2 & Q92945 & 1.7236 & 1.4466 & $\mathrm{sms} / \mathrm{smsdx}>\mathrm{ctrl}$ \\
\hline \multicolumn{6}{|l|}{ Signal transduction } \\
\hline Protein DJ-1 protein & PARK7 & Q99497 & -1.4112 & -1.5998 & $\operatorname{ctrl}>\operatorname{sms} / \mathrm{smsdx}$ \\
\hline Stathmin & STMN1 & P16949 & -1.3243 & -1.4821 & $\operatorname{ctrl}>\mathrm{sms} / \mathrm{smsdx}$ \\
\hline \multicolumn{6}{|l|}{ Structural molecular activity } \\
\hline Lamin B1 & LMNB1 & P20700 & 1.4199 & 1.7539 & sms/smsdx $>$ ctrl \\
\hline \multicolumn{6}{|l|}{ Translation } \\
\hline Eukaryotic translation initiation factor 5A-1 & IF5A1 & P63241 & -1.3835 & -1.6624 & $\operatorname{ctrl}>$ sms $/$ smsdx \\
\hline
\end{tabular}

Listed proteins have a fold change $<1,6$ and 'trend' indicate the direction of the change. Protein accession numbers from SwissProt/TrEMBL are given and the proteins are grouped according to molecular function. Smsdx and sms appear to have a similar effect demonstrated by the concordance in protein expression levels. Ctrl, control.

in the regulation of mitosis (33-35). High levels of stathmin expression were described in a variety of human malignancies, including prostate carcinoma (36). Prostate cancer is believed to provide one of the best models for the development of therapeutics that target stathmin (34). The level of expression of stathmin was shown to correlate with the malignant behaviour of prostate cancer cells. In fact, it was proposed that the level of expression of stathmin may serve as an important prognostic marker in prostate cancer. Mistry and Atweh (34) developed replication-deficient bicistronic adenoviral vectors that co-express green fluorescent protein and ribozymes that target stathmin mRNA. The therapeutic potential of these recombinant adenoviruses was tested in an experimental androgen-independent $\mathrm{LNCaP}$ prostate cancer model. Adenovirus-mediated transfer of anti-stathmin ribozymes resulted in efficient transduction and marked inhibition of stathmin expression in these cells.

The translationally controlled tumor protein (TCTP) was down-regulated by sms/smsdx. It is expressed in LNCaP and plays a role in controlling the apoptotic process (37). TCTP represents a novel anti-apoptotic protein involved in cell survival and apoptosis regulation (38) and may play a prominent role in the elongation cycle of translation (39). Accordingly, decreasing the expression of TCTP in cancer cells might be a way to decrease the efficiency of protein synthesis, and down-regulate cell proliferation. TCTP is also overexpressed in chemoresistant melanoma cell lines (40). This role in the process of tumor reversion might offer a lead to the identification of drugs that are able to diminish TCTP expression (41).

DJ-1 plays a direct role in the control of apoptosis in human prostate cells (42) and it was down-regulated by sms/ smsdx. The underexpression of DJ-1 results in decreased phosphorylation of $\mathrm{PKB} / \mathrm{Akt}$, while its overexpression leads to hyperphosphorylation of $\mathrm{PKB} / \mathrm{Akt}$ and increased cell survival. DJ-1 expression correlates negatively with PTEN immunoreactivity and positively with $\mathrm{PKB} / \mathrm{Akt}$ hyperphosphorylation. DJ-1 is thus a key negative regulator of PTEN that may be a useful prognostic marker for cancer (43). Treatment of cells from the human lung cancer cell line NCIH157 with paclitaxel and MEK inhibitor U0126 leads to a decrease in DJ-1 protein expression (44).

In conclusion, this analysis shows that sms and smsdx regulate a number of important proteins in the $\mathrm{LNCaP}$ prostatic cancer cell line. Several proteins were strongly regulated. The regulation was clearly concordant between sms and smsdx. Additionally, it was in general in agreement with the growth inhibitory actions of sms. The results support further clinical evaluation of smsdx and a phase II study on advanced prostate cancer patients is pending.

\section{Acknowledgements}

This study is supported by The Cancer Society in Stockholm, The King Gustav V Jubilee Fund, Stockholm and The Swedish Cancer Society.

\section{References}

1. Landis SH, Murray T, Bolden S and Wingo PA: Cancer statistics. CA Cancer J Clin 49: 8-31, 1999.

2. Crawford ED: Hormone refractory prostate cancer. Urology 54: S1-S7, 1999

3. Tannock IF, De Wit R, Berry WR, Horti J, Pluzanska A, Chi KN, Oudard S, Theodore C, James ND, Turesson I, Rosenthal MA and Eisenberger MA: TAX 327 Investigators docetaxel plus prednisone or mitoxantrone plus prednisone for advanced prostate cancer. N Engl J Med 351: 1502-1512, 2004. 
4. Sciarra A, Cardi A, Dattilo C, Mariotti G, Di Monaco F and Di Silverio F: New perspective in the management of neuroendocrine differentiation in prostate adenocarcinoma. Int $\mathrm{J}$ Clin Pract 60: 462-470, 2006

5. Woltering EA, Watson JC, Alperin-Lea RC, Sharma C, Keenan E, Kurozawa D and Barrie R: Somatostatin analogs: angiogenesis inhibitors with novel mechanisms of action. Invest New Drugs 15: 77-86, 1997.

6. Plonowski A, Schally AV, Nagy A, Sun B and Szepeshazi K: Inhibition of PC-3 human androgen-independent prostate cancer and its metastases by cytotoxic somatostatin analogue AN-238. Cancer Res 59: 1947-1953, 1999.

7. Sharma K and Srikant CB: Induction of wild-type p53, Bax, and acidic endonuclease during somatostatin-signaled apoptosis in MCF-7 human breast cancer cells. Int J Cancer 76: 259-266, 1998.

8. Behe M, Du J, Becker W, Behr T, Angerstein C, Marquez M, Hiltunen J, Nilsson S and Holmberg AR: Biodistribution, blood half-life, and receptor binding of a somatostatin-dextran conjugate. Med Oncol 18: 59-64, 2001.

9. Wulbrand U, Feldman M, Pfestroff A, Fehman HC, Du J, Hiltunen J, Marquez M, Arnold R, Westlin JE, Nilsson S and Holmberg AR: A novel somatostatin conjugate with a high affinity to all five somatostatin receptor subtypes. Cancer 94: 1293-1297, 2002.

10. Joensuu TK, Nilsson S, Holmberg AR, Marquez M, Tenhunen M, Saarto T and Joensuu H: Phase I trial on sms-D70 somatostatin analogue in advanced prostate and renal cell cancer. Ann NY Acad Sci 1028: 361-374, 2004.

11. Horoszewicz JS, Leong SS, Chu TM, Wajsman ZL, Friedman M, Papsidero L, Kim U, Chai LS, Kakati S, Arya SK and Sandberg AA: The LNCaP cell line - a new model for studies on human prostatic carcinoma. Prog Clin Biol Res 37: 115-132, 1980.

12. Langeler EG, van Uffelen CJ, Blankenstein MA, van Steenbrugge GJ and Mulder E: Effect of culture conditions on androgen sensitivity of the human prostatic cancer cell line LNCaP. Prostate 23: 213-223, 1993.

13. Lin MF, Garcia-Arenas R, Chao YC, Lai MM, Patel PC and Xia XZ: Regulation of prostatic acid phosphatase expression and secretion by androgen in LNCaP human prostate carcinoma cells. Arch Biochem Biophys 300: 384-390, 1993.

14. Lin MF, Lee MS, Garcia-Arenas R and Lin FF: Differential responsiveness of prostatic acid phosphatase and prostatespecific antigen mRNA to androgen in prostate cancer cells Cell Biol Int 24: 681-689, 2000.

15. Liu Z, Marquez M, Nilsson S, Holmberg AR and Alaiya AA: Proteomic analysis of a human prostate cancer cell line after incubation with a novel somatostatin 14 derivative. Cancer Genomics Proteomics 2: 347-352, 2005.

16. Brevini TA, Bianchi R and Motta M: Direct inhibitory effect of somatostatin on the growth of the human prostatic cancer cell line LNCaP: possible mechanism of action. J Clin Endocrinol Metab 77: 626-631, 1993.

17. Franzen B, Linder S, Okuzawa K, Kato H and Auer G: Nonenzymatic extraction of cells from clinical tumor material for analysis of gene expression by two-dimensional polyacrylamide gel electrophoresis. Electrophoresis 14: 1045-1053, 1993.

18. Ferjoux G, Lopez F, Esteve JP, Ferrand A, Vivier E, Vely F, Saint-Laurent N, Pradayrol L, Buscail L and Susini C: Critical role of Src and SHP-2 in sst2 somatostatin receptor-mediated activation of SHP-1 and inhibition of cell proliferation. Mol Biol Cell 14: 3911-3928, 2003

19. Kim R: Recent advances in understanding the cell death pathways activated by anticancer therapy. Cancer 103: 1551-1560, 2005.

20. Kroemer G and Reed JC: Mitochondrial control of cell death. Nat Med 6: 513-519, 2000.

21. Costantini P, Jacotot E, Decaudin D and Kroemer G: Mitochondrion as a novel target of anticancer chemotherapy. J Natl Cancer Inst 92: 1042-1053, 2000

22. Shimizu S, Narita $M$ and Tsujimoto $\mathrm{Y}$ : Bcl-2 family proteins regulate the release of apoptogenic cytochrome $\mathrm{c}$ by the mitochondrial channel VDAC. Nature 399: 483-487, 1999

23. Lawen A: Apoptosis: an introduction. Bioessays 25: 888-896, 2003.

24. Wood ZA, Schroder E, Harris J and Poole LB: Structure mechanism and regulation of peroxiredoxins. Trends Biochem Sci 28: 32-40, 2003.
25. Immenschuh $\mathrm{S}$ and Baumgart-Vogt E: Peroxiredoxins, oxidative stress, and cell proliferation. Antioxid Redox Signal 7: 768-777, 2005.

26. Baker AM, Oberley LW and Cohen MB: Expression of antioxidant enzymes in human prostatic adenocarcinoma. Prostate 32 229-233, 1997

27. Shen $C$ and Nathan C: Non-redundant antioxidant defense by multiple two-cysteine peroxiredoxins in human prostate cancer cells. Mol Med 8: 95-102, 2002.

28. Parcellier A, Gurbuxani S, Schmitt E, Solary E and Garrido C: Heat shock proteins, cellular chaperones that modulate mitochondrial cell death pathways. Biochem Biophys Res Commun 304: 505-512, 2003

29. Vargas-Roig LM, Gago FE, Tello O, Aznar JC and Ciocca DR Heat shock protein expression and drug resistance in breast cancer patients treated with induction chemotherapy. Int J Cancer 79: 468-475, 1998.

30. Misra UK, Gonzalez-Gronow M, Gawdi G, Hart JP, Johnson CE and Pizzo SV: The role of Grp 78 in alpha 2-macroglobulininduced signal transduction. Evidence from RNA interference that the low density lipoprotein receptor-related protein is associated with, but not necessary for, GRP 78-mediated signal transduction. J Biol Chem 277: 42082-42087, 2002.

31. Mintz PJ, Kim J, Do KA, Wang X, Zinner RG, Cristofanilli M, Arap MA, Hong WK, Troncoso P, Logothetis CJ, Pasqualini R and Arap W: Fingerprinting the circulating repertoire of antibodies from cancer patients. Nat Biotechnol 21: 57-63, 2003.

32. Arap MA, Lahdenranta J, Mintz PJ, Hajitou A, Sarkis AS, Arap W and Pasqualini R: Cell surface expression of the stress response chaperone GRP78 enables tumor targeting by circulating ligands. Cancer Cell 6: 275-284, 2004.

33. Marklund U, Larsson N, Gradin HM, Brattsand G and Gullberg M: Oncoprotein 18 is a phosphorylation-responsive regulator of microtubule dynamics. EMBO J 15: 5290-5298, 1996.

34. Mistry SJ and Atweh GF: Stathmin inhibition enhances okadaic acid-induced mitotic arrest: a potential role for stathmin in mitotic exit. J Biol Chem 276: 31209-31215, 2001.

35. Horwitz SB, Shen HJ, He L and Dittmar P: The microtubuledestabilizing activity of metablastin (p19) is controlled by phosphorylation. J Biol Chem 272: 8129-8132, 1997.

36. Friedrich B, Gronberg H, Landstrom M, Gullberg M and Bergh A Differentiation-stage specific expression of oncoprotein 18 in human and rat prostatic adenocarcinoma. Prostate 27: 102-109, 1995.

37. Arcuri F, Papa S, Carducci A, Romagnoli R, Liberatori S, Riparbelli MG, Sanchez JC, Tosi P and del Vecchio MT: Translationally controlled tumor protein (TCTP) in the human prostate and prostate cancer cells: expression, distribution, and calcium binding activity. Prostate 60: 130-140, 2004.

38. Li F, Zhang D and Fujise K: Characterization of fortilin, a novel antiapoptotic protein. J Biol Chem 276: 47542-47549, 2001.

39. Cans C, Passer BJ, Shalak V, Nancy-Portebois V, Crible V, Amzallag N, Allanic D, Tufino R, Argentini M, Moras D, Fiucci G, Goud B, Mirande M, Amson R and Telerman A: Translationally controlled tumor protein acts as a guanine nucleotide dissociation inhibitor on the translation elongation factor eEF1A. Proc Natl Acad Sci USA 100: 13892-13897, 2003

40. Sinha P, Kohl S, Fischer J, Hutter G, Kern M, Kottgen E, Dietel M, Lage H, Schnolzer M and Schadendorf D: Identification of novel proteins associated with the development of chemoresistance in malignant melanoma using two-dimensional electrophoresis. Electrophoresis 21: 3048-3057, 2000.

41. Tuynder M, Fiucci G, Prieur S, Lespagnol A, Geant A, Beaucourt S, Duflaut D, Besse S, Susini L, Cavarelli J, Moras D, Amson R and Telerman A: Translationally controlled tumor protein is a target of tumor reversion. Proc Natl Acad Sci USA 101: 15364-15369, 2004

42. Kim RH, Peters M, Jang Y, Shi W, Pintilie M, Fletcher GC, De Luca C, Liepa J, Zhou L, Snow B, Binari RC, Manoukian AS, Bray MR, Liu FF, Tsao MS and Mak TW: DJ-1, a novel regulator of the tumor suppressor PTEN. Cancer Cell 7: 263-273, 2005.

43. MacKeigan JP, Clements CM, Lich JD, Pope RM, Hod Y and Ting JP: Proteomic profiling drug-induced apoptosis in nonsmall cell lung carcinoma: identification of RS/DJ-1 and RhoGDIalpha. Cancer Res 63: 6928-6934, 2003.

44. Hod Y: Differential control of apoptosis by DJ-1 in prostate benign and cancer cells. J Cell Biochem 92: 1221-1233, 2004. 